\title{
The Stratospheric Aerosol and Gas Experiment (SAGE III) on the International Space Station (ISS) Mission Michael Cisewski ${ }^{\mathrm{a}}$, Joseph Zawodny ${ }^{\mathrm{a}}$, Joseph Gasbarre ${ }^{\mathrm{a}}$, Richard Eckman ${ }^{\mathrm{b}}$, Nandkishore Topiwala ${ }^{\mathrm{b}}$, Otilia Rodriguez-Alvarez ${ }^{\mathrm{c}}$, Dianne Cheek $^{\mathrm{a}}$, Steve Hall $^{\mathrm{a}}$ \\ ${ }^{a}$ NASA Langley Research Center, 11 Langley Blvd, Hampton, VA, USA 23681 \\ ${ }^{b}$ NASA Headquarters, 300 E Street Southwest, Washington, DC 20546 \\ ${ }^{\mathrm{c}}$ NASA Goddard Space Flight Center, 8800 Greenbelt Rd, Greenbelt, MD 20771
}

\begin{abstract}
The Stratospheric Aerosol and Gas Experiment III on the International Space Station (SAGE III/ISS) mission will provide the science community with high-vertical resolution and nearly global observations of ozone, aerosols, water vapor, nitrogen dioxide, and other trace gas species in the stratosphere and upper-troposphere. SAGE III/ISS measurements will extend the long-term Stratospheric Aerosol Measurement (SAM) and SAGE data record begun in the 1970s. The multi-decadal SAGE ozone and aerosol data sets have undergone intense scrutiny and are considered the international standard for accuracy and stability. SAGE data have been used to monitor the effectiveness of the Montreal Protocol. Key objectives of the mission are to assess the state of the recovery in the distribution of ozone, to re-establish the aerosol measurements needed by both climate and ozone models, and to gain further insight into key processes contributing to ozone and aerosol variability. The space station mid-inclination orbit allows for a large range in latitude sampling and nearly continuous communications with payloads.

The SAGE III instrument is the fifth in a series of instruments developed for monitoring atmospheric constituents with high vertical resolution. The SAGE III instrument is a moderate resolution spectrometer covering wavelengths from 290 $\mathrm{nm}$ to $1550 \mathrm{~nm}$. Science data is collected in solar occultation mode, lunar occultation mode, and limb scatter measurement mode.

A SpaceX Falcon 9 launch vehicle will provide access to space. Mounted in the unpressurized section of the Dragon trunk, SAGE III will be robotically removed from the Dragon and installed on the space station. SAGE III/ISS will be mounted to the ExPRESS Logistics Carrier-4 (ELC-4) location on the starboard side of the station. To facilitate a nadir view from this location, a Nadir Viewing Platform (NVP) payload was developed which mounts between the carrier and the SAGE III Instrument Payload (IP).
\end{abstract}

Keywords: Stratosphere, Ozone, Aerosol, solar occultation, lunar occultation, International Space Station

\section{INTRODUCTION}

SAGE III/ISS will extend the SAGE data record from the ideal vantage point of the International Space Station. The space station orbital inclination of $51.6^{\circ}$ is ideal for SAGE measurements providing coverage between $70^{\circ}$ north and $70^{\circ}$ south latitude. The SAGE data record includes an extensively validated data set including aerosol optical depth data dating to the Stratospheric Aerosol Measurement (SAM) ${ }^{1}$ experiments in 1975 and 1978 and stratospheric ozone profile data dating to the Stratospheric Aerosol and Gas Experiment (SAGE) in 1979. These and subsequent data records, notably from the SAGE II $^{2}$ experiment launched on the Earth Radiation Budget Satellite in 1984 and the SAGE III $^{3}$ experiment launched on the Russian Meteor-3M satellite in 2001, have supported a robust, long-term assessment of key atmospheric constituents.

The mission architecture heavily leverages the existing ground network in place for the ISS and previous SAM and SAGE missions. SAGE III/ISS operations are conducted by an operations team based at NASA's Langley Research Center through the ISS Payload Operations Integration Center (POIC) at NASA's Marshall Space Flight Center. The POIC integrates NASA payload operations and provides a gateway for payload command and data transfer to the ISS. The SAGE III team, using systems housed at NASA's Langley Atmospheric Sciences Data Center (ASDC), produces SAGE III science data products. The data products are produced using a common SAGE III code base. The ASDC is responsible for archival of the data and distribution to the larger science community. 


\section{SAGE III/ISS Science Objectives}

The primary objective of SAGE III/ISS is to monitor the vertical distribution of aerosols, ozone, and other trace gases in the Earth's stratosphere and troposphere to enhance the understanding of ozone recovery and climate change processes in the upper atmosphere. SAGE III/ISS measurement requirements are summarized in Table 1.0 below.

Table 1-0: Baseline Data Products

\begin{tabular}{|l|l|l|l|l|l|}
\hline Product & Description & $\begin{array}{l}\text { Measurement } \\
\text { Type }\end{array}$ & $\begin{array}{l}\text { Vertical } \\
\text { Range } \\
(\text { Km })\end{array}$ & $\begin{array}{l}\text { Vertical } \\
\text { Resolution } \\
(\mathrm{Km})\end{array}$ & $\begin{array}{l}\text { Precision } \\
0 *-100\end{array}$ \\
\hline 1 & Level 1B Transmissions & Solar & 0.75 & $0.1 \% * *$ \\
\hline 2 & Aerosol Extinction Coefficients & Solar & $0 *-40$ & 0.75 & $5 \%$ \\
\hline 3 & Aerosol Optical Depth & Solar & N/A & N/A & $5 \%$ \\
\hline 4 & Ozone (O3) Concentration & Solar & $0 *-50$ & 0.75 & $5 \%$ \\
\hline 5 & Water Vapor (H2O) Concentration & Solar & $5 *-45$ & 0.75 & $10 \%$ \\
\hline 7 & Nitrogen Dioxide (NO2) Concentration & Solar & TP+2 - 45 & 0.75 & $10 \%$ \\
\hline
\end{tabular}

Notes: *or cloud-top altitude, $* *$ defined at $600 \mathrm{~nm}, \mathrm{TP}=$ altitude of the tropopause

In addition to these baseline products, SAGE III/ISS will produce a number of products in research mode - notably limb scatter radiance measurements during the day-lit portion of the orbit and Lunar Occultation Nitrogen Dioxide (NO2) and Nitrogen Trioxide (NO3) Concentration measurements.

SAGE III/ISS will meet these measurement objectives by making measurements using the solar occultation, lunar occultation, and limb scatter measurement techniques. Solar occultation measurements use the sun as a light source; the instrument measures the solar spectrum un-attenuated by the atmosphere during each sunrise or sunset measurement. By using this technique, each measurement is essentially self-calibrated, eliminating long-term changes in instrument performance as a significant error source. In addition to the traditional solar measurements, SAGE III will also obtain data via lunar occultation and via the limb scattering technique. Anticipated solar and lunar measurement coverage is shown in Figure 1.

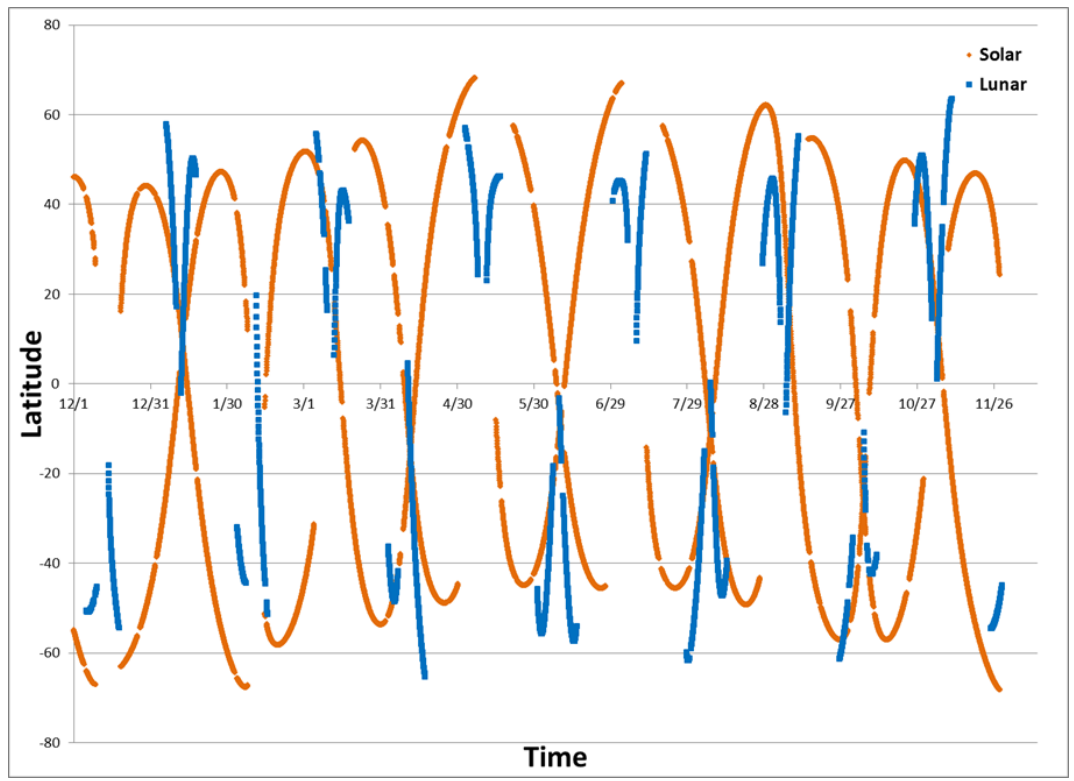

Figure 1: Solar and Lunar Measurement Coverage 


\section{SAGE III/ISS Mission Concept}

The SAGE III team worked closely with the ISS program to develop the SAGE III/ISS mission concept. The mission concept leverages the capabilities of the ISS and the Expedite the Processing of Experiments to the Space Station (ExPRESS) pallet for attached payloads. Because ExPRESS pallet-attached payload requirements are well defined, the SAGE III team was able to focus design efforts on finding the optimal location for making SAGE III measurements from the ISS and developing the payload systems necessary to support the science measurements.

As in home real estate, in science there is no substitute for location. Extensive trade studies were conducted to find the ideal mounting location for the SAGE III/ISS payload. Because the science measurements require unobstructed views of the limb of the Earth through solar beta angles of $\pm 60^{\circ}$, efforts were focused on identifying a nadir viewing ExPRESS Logistics Carrier (ELC) site with minimal obstructions from other ISS components. The best sites were found on ELC-4 and on the European Space Agency Columbus module. The ELC-4 site was chosen because other payloads were planning on utilizing the Columbus site and because of potentially more complex avionics interfaces. The ELC-4 site, shown in Figure 2, was selected in conjunction with the ISS program and is optimum location for SAGE-type measurements on the ISS.

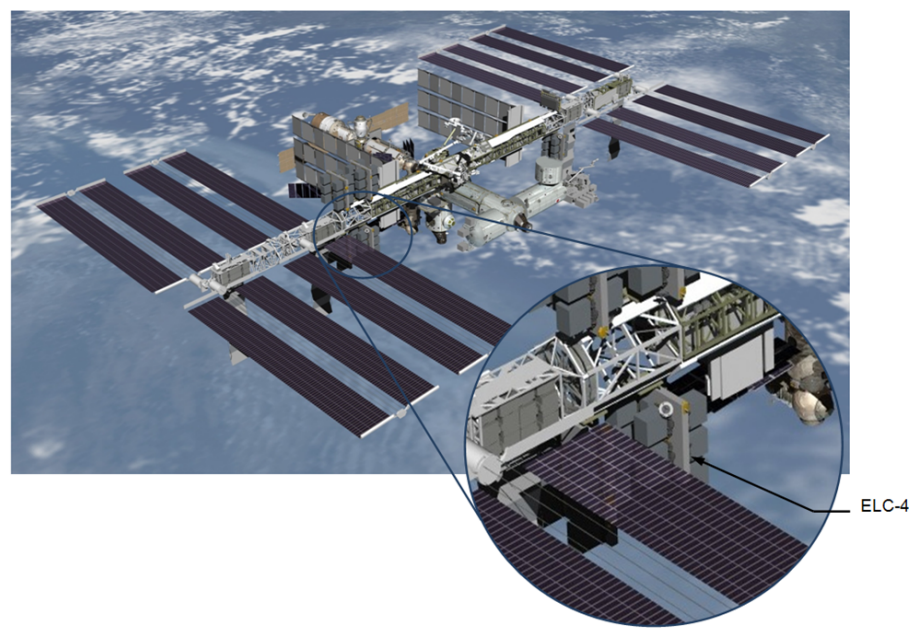

Figure 2: $\quad$ SAGE III/ ISS Mounting Location on ELC-4

While the ELC-4 site was ideal, packaging the SAGE III payload to meet the science objectives while meeting envelope requirements remained a challenge. A novel Nadir Viewing Platform (NVP) concept solved the packaging problems. The concept involves using the NVP as a separate ExPRESS pallet payload to translate the ExPRESS pallet interface by $90^{\circ}$ fully replicating the mechanical and electrical interfaces and providing the required nadir orientation. The resulting payload concept with the NVP and SAGE III Instrument Payload (IP) integrated on the ELC-4 is shown in Figure 3.

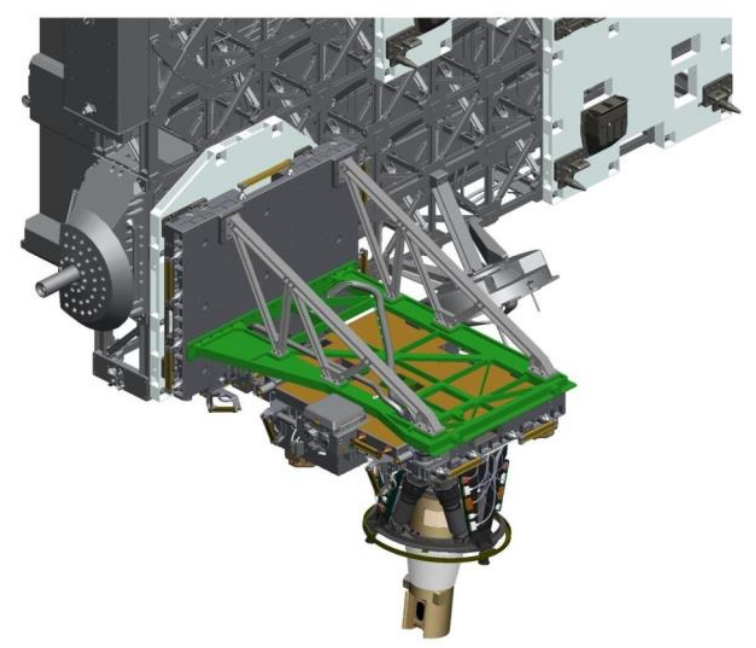

Figure 3: SAGE III/ISS Payload on ELC-4 
SAGE III/ISS will be transported to the space station using the SpaceX Falcon 9 launch vehicle. Mounted using the ExPRESS Payload Adapter (ExPA) pallet to the Falcon 9 Dragon trunk section, the SAGE III IP and the NVP along with another payload will be launched to the ISS. Launch is planned on the SpaceX-10 flight in February 2016. The Dragon trunk with the SAGE III IP and NVP are shown in Figure 4.

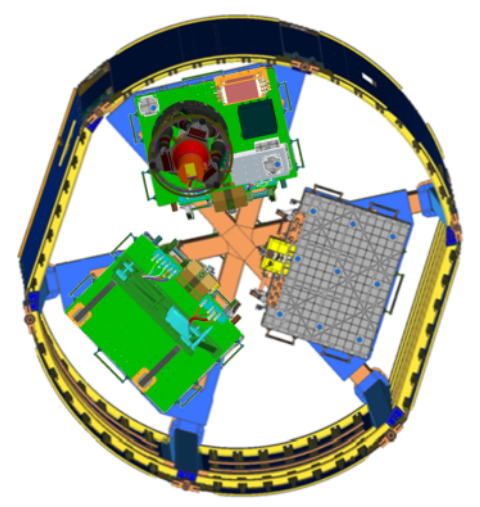

Figure 4: SAGE III Mounted in Dragon Trunk Section

Once the SpaceX Dragon berths with the ISS, SAGE III will be removed from the trunk section and installed to the ELC mounting location using ISS robotics. The MAGIK robotic analysis team at NASA's Johnson Space Flight Center developed the installation sequence. First, the NVP and SAGE III IP are removed from the Dragon Trunk using the Dextre robot, or Special Purpose Dexterous Manipulator (SPDM). The NVP and SAGE III IP are temporarily stored on the Enhanced ORU Temporary Platform (EOTP). After transport to the ELC-4 site, the NVP is installed to the ELC and the SAGE III IP is installed to the NVP.

\section{SAGE III Instrument Payload}

The SAGE III / ISS Instrument Payload (IP) is based on an existing SAGE III flight instrument and an existing European Space Agency (ESA) provided Hexapod course pointing system, both originally developed for flight on the ISS as attached payloads beginning in mid-1990's. The SAGE III instrument and Hexapod are part of the SAGE III/ISS IP. Other SAGE III/ISS IP components include the robotically installable ExPRESS Payload Adapter (ExPA), a new avionics unit called the Interface Adapter Module (IAM), two Contamination Monitoring Packages (CMP), and a Disturbance Monitoring Package (DMP). The SAGE III/ISS IP is shown in Figure 5.

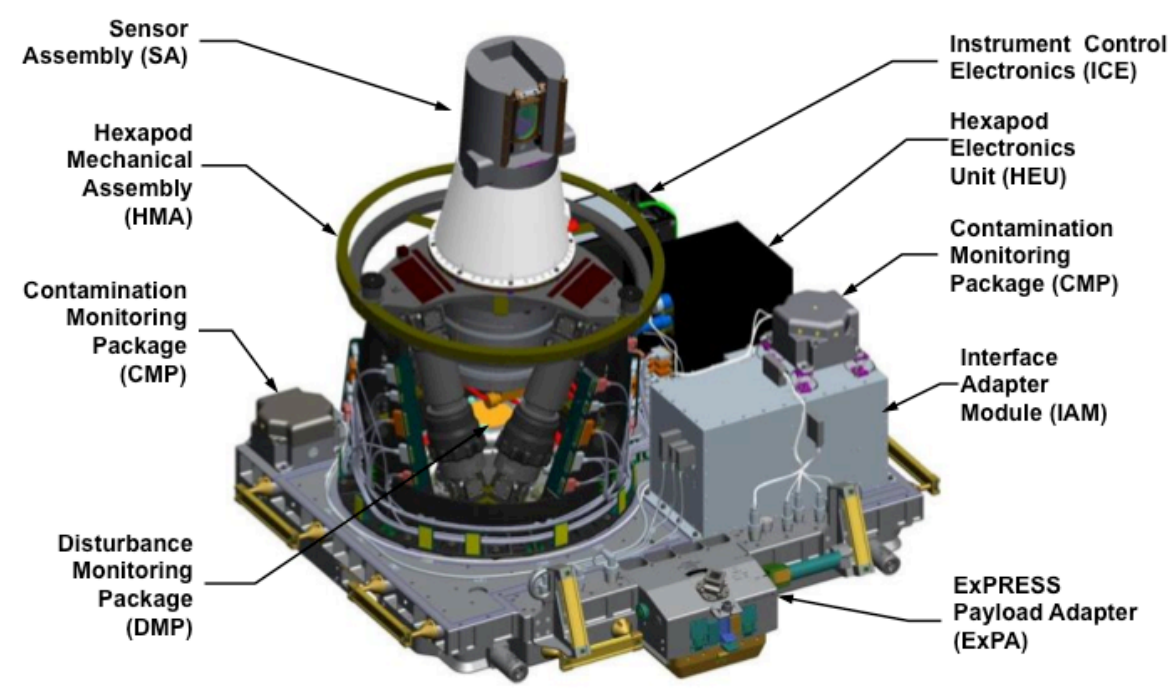

Figure 5: SAGE III/ISS Instrument Payload (IP) 
The SAGE III instrument consists of the Sensor Assembly (SA) and the Instrument Control Electronics (ICE). The SAGE III instrument is a moderate resolution spectrometer covering wavelengths from 290 to 1550 nanometers (nm). In addition to the spectrometer, the instrument includes an azimuth gimbal and tracking system to capture and track the sun or the moon and an elevation scanning system used to scan the sun or the moon. Ball Aerospace and Technology Corporation (BATC) was the SAGE III instrument prime contractor and has supported the refurbishment of the instrument for the current mission performed at NASA's Langley Research Center.

The Hexapod Pointing System consists of the Hexapod Mechanical Assembly and the Hexapod Electronics Unit. To accommodate changes in the ISS attitude, the Hexapod will orient the SAGE III Sensor Assembly in a nadir orientation prior to each solar observation and lunar observation. The Hexapod can accommodate attitude variations of up to range $\pm 8^{\circ}$ from its mounting base which is wedged $-7^{\circ}$ in the ISS ram $(+X)$ direction to closely match the nominal ISS torque equilibrium attitude. ESA developed the Hexapod under contract to Thales Alenia Space-Italy (TAS-I) in Turin, Italy.

Two Contamination Monitoring Packages (CMPs) are used to monitor the contamination environment on the ISS and support operational decision-making. One CMP has five (5) sensors and the other CMP has three (3) sensors. Together, the sensors provide $360^{\circ}$ coverage with overlapping fields of view. The CMPs are based on Thermoelectric Quartz Crystal Microbalances (TQCMs) manufactured by QCM Research with electronics and packaging developed by NASA’s Langley Research Center.

Because the SAGE III instrument optical path is susceptible to molecular contamination degradation, SAGE instrument operations personnel will use CMP data to determine if science operations should be conducted with the contamination door open or the contamination door closed (science operations are possible because the contamination door contains an optical grade window). While science operations with the contamination door open are preferred, closed-door operations will enable additional data collection with acceptable risk during periods of visiting vehicle traffic or when new ISS hardware is being added and is outgassing at elevated rates. CMP data makes sound operational decisions possible.

A Disturbance Monitoring Package (DMP) is used to capture small variations in ISS attitude to support science instrument data analyses and correct for variations in the line-of-sight from the instrument to the sun or the moon if necessary. The DMP is a Commercial-Off-The-Shelf (COTS) Miniature Inertial Reference Unit (MIMU) manufactured by the Honeywell Corporation.

An Interface Adapter Module (IAM) is used to make the existing payload elements, new payload elements, and the ISS work together seamlessly. The IAM conditions, controls, and distributes 28 -volt power and 120 -volt power via an internal IAM Power Distribution Unit (PDU). The IAM manages MIL-1553B communications between internal payload elements (SAGE III instrument, Hexapod, and the DMP) functioning as a MIL-1553B bus controller. The IAM communicates with ISS per ISS Command \& Data Handling (C\&DH) protocols functioning as a MIL-1553B remote terminal. The IAM manages RS-422 communications between internal payload elements (PDU, CMPs, and DMP). The IAM stores all payload data on an 8-gigabyte solid-state recorder capable of holding a minimum of three days of science observations. The solid-state recorder is transmitted to the ISS over a rad-hardened Ethernet 10Base-T interface.

All of these functions are controlled via the IAM Power PC-based on-board computer. In addition to managing the communications and control tasks previously described, the IAM manages absolute time commands, relative time sequence commands, and performs either manual or autonomous on-board science measurement scheduling. The IAM provides Fault Detection Isolation and Recovery (FDIR) functionality to continually monitor payload internal system health and safe the system if a problem is detected.

The IAM flight hardware is a combination of COTS on-board computer components developed by Aitech and in-house designs for the PDU and the solid-state recorder. NASA Langley developed the IAM chassis and performed system integration and qualification. Flight software was written primarily by NASA Langley in collaboration with BATC. The software utilizes the VX works real-time operating system and leverages the stable and proven CALIPSO mission code base.

The final payload element is the ExPRESS Payload Adapter or ExPA. The ExPA, provided to the Project by the ISS Program, is the mounting structure used to support payload integration and is compatible with both launch vehicle and on-orbit interfaces. The ExPA includes provisions for Extra Vehicular Robotics necessary for installation to the ISS and includes blind mate connectors necessary for power and avionics. 


\section{Nadir Viewing Platform}

The Nadir Viewing Platform was designed to translate an existing payload site on the ISS ExPRESS Logistics Carrier-4 (ELC-4) to a nadir viewing payload site. Like the SAGE III Instrument Payload, the NVP utilizes an ExPA as a mounting structure and a truss structure to replicate the ELC mechanical interface. Electrically, the NVP replicates the existing electrical interface. The NVP is shown in Figure 6.

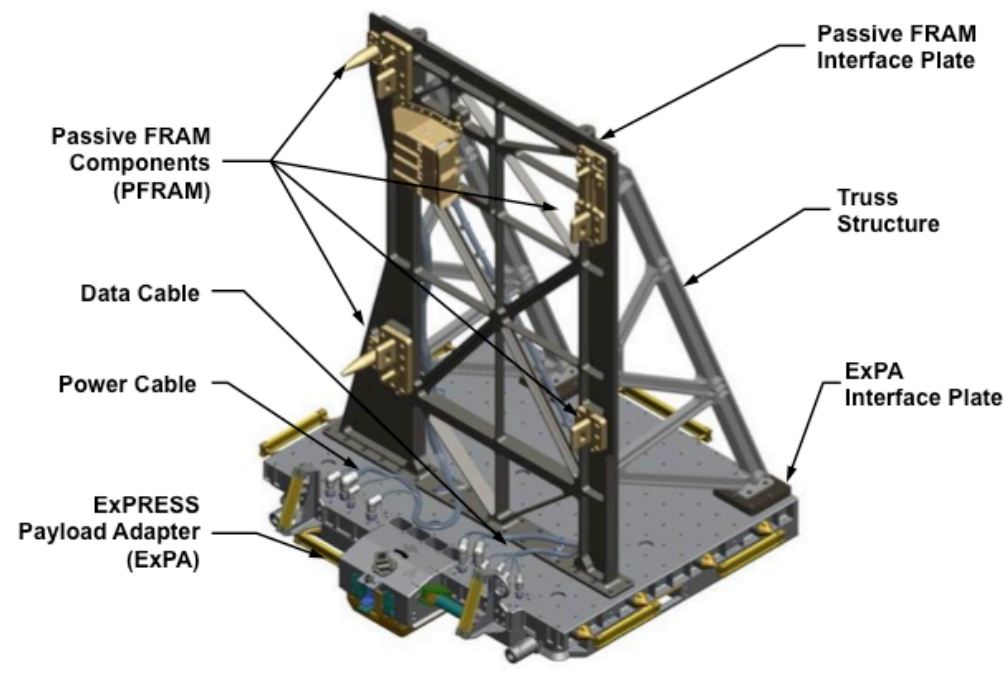

Figure 6: Nadir Viewing Platform

\section{Mission Operations and Science Data Processing}

The SAGE III/ISS mission architecture integrates the mission segments to ensure successful execution. Figure 7 illustrates the following Mission Architecture features.

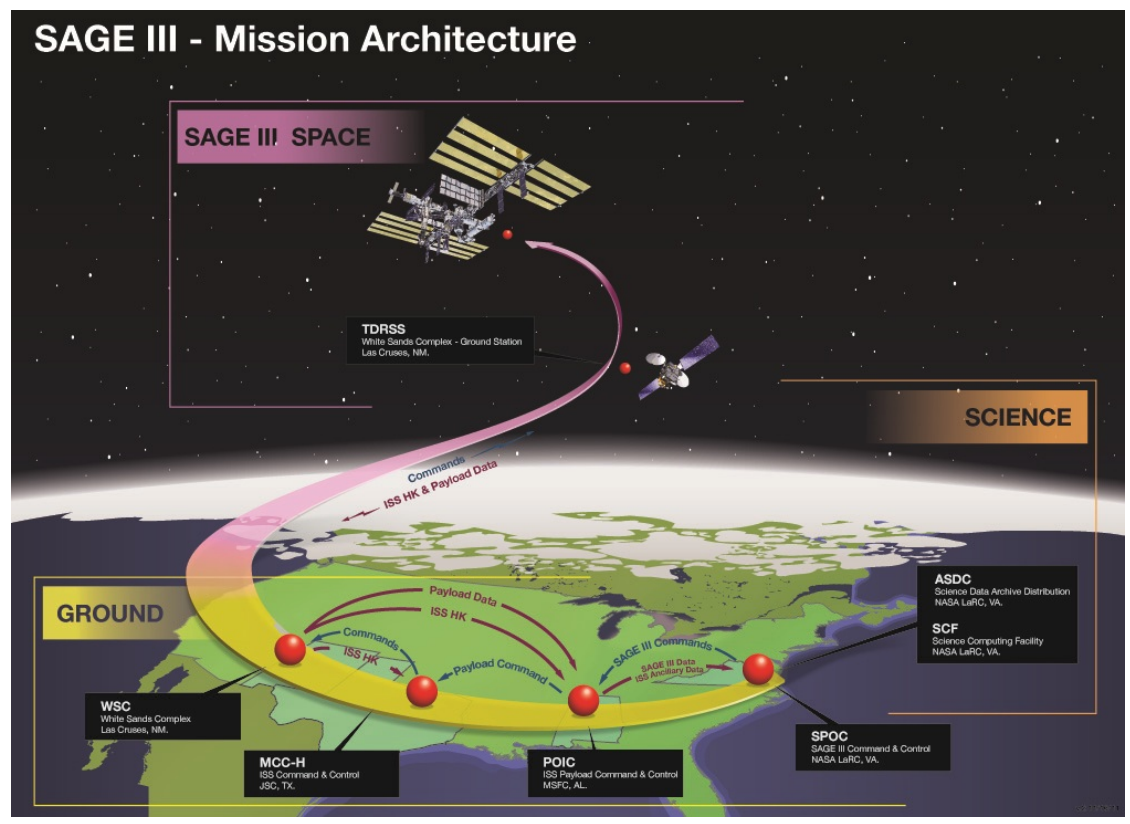

Figure 7: SAGE III Mission Architecture

Key features of the mission architecture include use of the existing infrastructure in place to support ISS operations. This infrastructure includes the Tracking and Data Relay Satellite System (TDRSS), which transmits data from ISS to 
the White Sands Complex (WSC), the Mission Control Center Houston (MCC-H) at Johnson Space Center (JSC) where the American Segment of ISS is managed, and the Payload Operations Integration Center (POIC) at Marshall Space Flight Center (MSFC) where all NASA payloads on ISS are managed.

The SAGE III Payload Operations Center (SPOC) located at NASA's Langley Research Center (LaRC) manages and operates the SAGE III Instrument Payload. The SPOC utilizes tools developed and used during payload development to operate the payload. The SPOC will be staffed five days per week / 8 hours per day during routine operations. Nominal payload stored commands will be transmitted weekly through interfaces with the POIC. Ground controllers at the POIC monitor SAGE III payload health and status $24 / 7$ and will contact the SPOC team if any problems are detected. Engineering telemetry is also monitored autonomously by SPOC systems and the system has been designed to issue alerts to on-call team members if a problem is detected. In addition to the autonomous checks, SPOC team members perform operational status checks at least twice daily. SAGE III science data is stored on an on-board recorder that will be downloaded once daily.

The SPOC will configure the payload in a safe state for special ISS operations such as vehicles arriving or departing the ISS, orbit maintenance maneuvers, ISS hardware configuration changes, and Extra Vehicular Activities. Operations are conducted to ensure crew safety, maintain SAGE III payload capabilities, and to maximize science data collection.

SAGE III science data is produced at the Science Computing Facility (SCF) located at NASA's Langley Research Center. Recorded telemetry, ISS definitive ephemeris and GEOS5 meteorological data are necessary to produce SAGE III science data. Science data products are produced using a common code base used for previous SAGE missions with necessary adaptations for the ISS mission.

SAGE III science data products are archived and distributed by the Atmospheric Sciences Data Center (ASDC) located at NASA's Langley Research Center. Data products will be available for the science community six (6) months after the initial on-orbit checkout period and with a latency not to exceed fourteen days after nominal science data production has begun.

\section{Summary}

SAGE III/ISS will extend the SAGE data record from the ideal vantage point of the ISS. SAGE III measurements will provide the scientific community with data on key atmospheric constituents necessary to monitor the health of our planet.

\section{Acknowledgement}

The authors of this paper would like to acknowledge the outstanding efforts of an incredibly talented international team working to make this mission a success.

*m.s.cisewski@nasa.gov phone 1757 864-1861; http://sage.nasa.gov/SAGE3ISS/

${ }^{1}$ Chu, W. P. and M. P. McCormick, Inversion of Stratospheric Aerosol and Gaseous Constituents From Spacecraft Solar Extinction Data in the 0.38-1.0-micron Wavelength Region, Appl. Opt., 18, no. 9, 1404-1413

${ }^{2}$ Mauldin, L. E., N. Zaun, M. McCormick, J. Guy, W. Vaughn, Stratospheric Aerosol And Gas Experiment II Instrument: A Functional Description, Optical Engineering 24(2), 242307, April 1, 1985

${ }^{3}$ Mauldin, L. E., Salikhov, R., Habib, S., Vladimirov, A.; Carraway, D., Petrenko, G., Comella, J.; Meteor3M(1)/Stratospheric Aerosol and Gas Experiment III (SAGE III) Jointly Sponsored by the National Aeronautics and Space Administration and the Russian Space Agency; SPIE Vol. 3501・0277-786X198 